\title{
DAMAGE DETECTION OF THE ROD IN THE CROSSFLOW USING SURROGATE-BASED MODELLING
}

\author{
S. Upnere ${ }^{1,2, *}$, J. Auzins ${ }^{1}$ \\ ${ }^{1}$ Institute of Mechanics and Mechanical Engineering, \\ Riga Technical University, 6B Kipsalas Str., Riga, LATVIA \\ ${ }^{2}$ Ventspils University of Applied Sciences, \\ 101 Inzenieru Str., Ventspils, LATVIA \\ *e-mail: upnere@protonmail.com
}

An effective and accurate methodology is developed to create an inverse surrogate model for the mass reduction analysis of the rod in the rod bundle inserted in the crossflow. The performance of two surrogate modelling approaches has been evaluated. These models are the Response Surface Method and Legendre polynomial approximations. The relationship between dominant frequencies, support stiffness and rod mass derived from Computational Fluid Dynamics simulations is used as input data for approximations. The selection of sample points is implemented with a new type of orthogonal design. The results have shown that the proposed methodology can reliably replace the finite volume model and drastically reduce computational time.

Keywords: CFD, inverse model, rod bundle, surrogate model.

\section{INTRODUCTION}

For the safe operation of engineering equipment, it is necessary to continuously monitor whether the system does not exceed its operational limits and does not compromise its structural integrity. Relatively frequently it is not possible to measure changes in the internal elements of the system directly during the operation of the equipment, for example, in heat exchangers or nuclear reactor assembly. For complex systems, their behaviour as a function of the input parameters is generally not known. In such situations, physical and numerical experiments can often be the only way to 
determine the relevant relationships. Nevertheless, full-model simulations would require long calculations from several hours to several days or weeks, even using highperformance computing resources. That approach is not applicable to the monitoring of the system during its operation. Therefore, to reduce computing time, simplified models - surrogate models or metamodels - are used that approximate the original model. Adequate mathematical models can be used to detect as early as possible changes in the typical behaviour of the system that could indicate its malfunction and structural integrity problems.

This study focuses on heat exchangers consisting of a bundle of rods placed in a highly turbulent crossflow. Flow induces rod vibrations with a typical spectrum that can be used to indirectly detect changes in system parameters. The decrease in the mass of the rod may be one of the parameters indicating that damage has occurred in the installation. Therefore, Computational Fluid Dynamics (CFD) simulations and surrogate modelling approaches are used to find the relationship between the mass of the rod and the dominant frequencies.

In the literature, various types of engineering problems are solved through surrogate models such as design optimization, uncertainty quantification, parameter identification, sensitivity analysis or inverse problems. Available surrogate models include, e.g., Response Surface Method (RSM), Kriging (or Gaussian process modelling), radial basis functions and artificial neural networks. RSM is predicated on the assumption that many physical systems are smooth and continuous and can be well approximated, at least in the region of interest, by low-order polynomials [1]. As follows from [2], the polynomial approximation is not applicable to highly non-linear functions, but in cases of low non-linearity and low dimensions, it is an effective approach. The Kriging method is of most use when the true function is particularly computationally intensive, e.g., a CFD-based calculation [3]. As an example, we can mention the minimization of vortexinduced vibrations using Kriging described by Filho et al. [4]. Banyay et al. [5], [6] used Kriging surrogate for global sensitivity analysis of flow-induced vibrations. In the present paper, low-order (less than 4th order) polynomials and Legendre polynomial were investigated. Two-factor forward and inverse surrogate models were created.

The training data set for the surrogate model development is generated by exercising complex high accuracy models such as finite volume or finite element models. The selection of sample points can be implemented through the design of experiments. The design of experiments is very important for a high-quality surrogate model. There are several metrics to determine the adequacy and goodness of fit of the model, such as a root mean square error, cross-validation error, T-tests or Pearson's $\chi^{2}$ test, etc. As mentioned by Greenwood and Nikulin [7], when the amount of data is small, no test is very good, and when there are a lot of high-quality data almost any test, properly designed and applied, will give good results. However, when addressing problems with high computational costs, an effective sampling plan must be sought, which means a minimum number of points that provide a surrogate model with good precision. Techniques that are commonly considered are Latin Hypercube Sampling, Central Composite Design (CCD), orthogonal arrays, factorial designs. In this study, a new type of orthogonal design [8] is used. Based on the obtained sample points, the selection of initial conditions for the fullorder model is carried out.

The paper is structured as follows. In 
Section 2, the CFD approach applied to obtain the results of the full model is formulated. Section 3 describes the types of employed surrogate models, the sampling method and the model accuracy metrics. Verification results are presented in Section 4. Finally, concluding remarks are given in Section 5.

\section{HIGH-FIDELITY COMPUTATION}

The full model uses the configuration of the rod bundle described in Upnere et al. [9], in which laboratory experiments were carried out to examine the vibrations of single flexible-mounted rod caused by the crossflow in the rigid rod array. The configuration has a triangular array with pitchto-diameter ratio, $P / d=1.1$. The rod diameter is $8 \mathrm{~mm}$ and it is made of steel (in the laboratory experiment).
The open-source CFD tool OpenFOAM 2.4.x was used to solve differential equations of mass and momentum. Unsteady Reynolds-Averaged Navier-Stokes (URANS) equations are solved using the $k$ - $\omega$ SST turbulence model to ensure the closure of the equation system. A lowReynolds turbulence model approach was used. The $2 \mathrm{D}$ problem was addressed to simplify numerical calculations.

\subsection{Description of the Numerical Model}

The investigation of an appropriate computational domain was achieved through a decoupling approach. The number of cylinders was reduced by columns in the in-flow direction and by rows in the crossflow direction. Several computational domains were analysed to find an optimal case given both the accuracy of the results and the cost of computing. The columns and rows of the cylinder arrays were decreased step by step with a decrement 1 . Other conditions remained the same. The drag force $F_{x}$ was used to compare the impact of array rows and columns.

The largest analysed domain was a $4 \times 5$ cylinder array, see the domain A in Fig. 1. Decreasing rows from four to two changes the force $F_{x}$ by less than $1 \%$. The boundary condition at the top and bottom is simplified as a symmetry condition. The reduction of columns in the direction of flow by removing one column behind the test cylinder causes a non-significant change in the calculated force. In contrast, the reduction of one column in the direction of upstream causes a change of approximately $4 \%$. Because of the above, the domain B (see Fig. 1) can be considered an optimal computational domain for the analysed Reynolds numbers and array configuration.
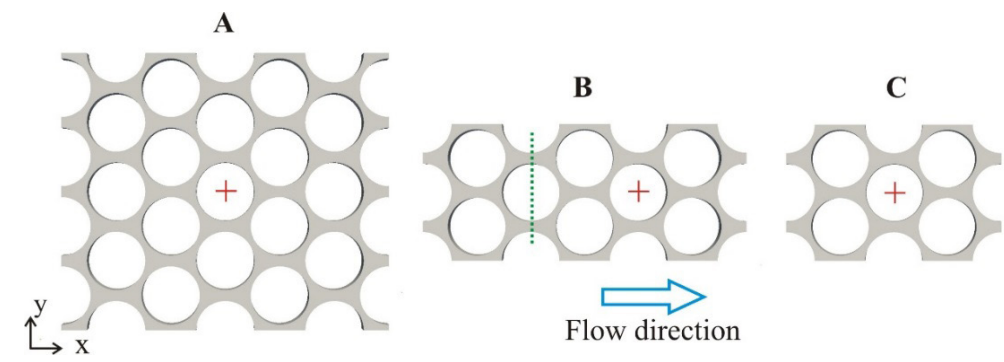

Fig. 1. Analysed computational domains. The centre of the test cylinder is marked with the red cross. 
As RANS-type calculations are performed that are time-averaged, it is possible to determine a typical distribution of flow between two adjacent cylinders, see the green dotted line in the domain B. Using the found flow profile as an inflow boundary condition, the computational domain can be reduced to a $2 \times 2$ array of cylinders, see the domain $\mathrm{C}$ in Fig. 1. This approach is useful because the developed surrogate models require a set of calculations that change only the mass and support stiffness of the test cylinder and not the characteristic of the inlet flow. Operating pressure $p=0 \mathrm{~Pa}$ was defined on the outflow boundary.

Different mesh refinements were studied to avoid the influence of the cell size on the numerical simulations. As a low-Reyn- olds turbulence model was used, particular attention was paid to the correct description of the boundary layer around cylinders. The final version of the mesh contains 476000 cell elements. Around cylinders, it contains 25 layers with an expansion ratio of 1.05 , providing $\mathrm{y}^{+}$less than 1 .

Based on studies available in the literature, it is known that the largest oscillations are in a direction perpendicular to the flow and therefore the movement of the test cylinder is only allowed in the transverse direction. The rotation of the test cylinder is also not allowed. The movement of the test cylinder in crossflow was studied by mounting it as a massspring system, where $m$ is the mass of the oscillating part and $k$ is the stiffness coefficient of the mounting.

\subsection{Verification and Validation of the Model}

According to Roache [10], the quantification of uncertainty in numerical simulations contains the three most important items: verification of codes, verification of calculations and validation. Since standard OpenFOAM solvers are used in the modelling process, the verification of the code is not carried out in this case. Solu- tion verification is carried out using flow simulation around a single cylinder at $\mathrm{Re}=$ 11000 and comparing integral parameters with data available in the literature. Table 1 shows a summary of the drag coefficient, $C_{d}=2 F_{x} /\left(u^{2} S\right)$, where $u$ is flow velocity, $S$ is an area that interacts with the flow and Strouhal number, $S t$, from the various studies.

Table 1. Comparison of the Modelled and Experimental Results of the Drag Coefficient $C_{d}$ and Strouhal Number $S t$

\begin{tabular}{|l|c|c|}
\hline The study & $C_{d}$ & $S t$ \\
\hline Khan et al. [11], RANS, 2D & 1.150 & 0.201 \\
\hline Khan et al. [11], RANS, 3D & 1.210 & 0.203 \\
\hline Dong et al. [12], DNS & 1.208 & 0.209 \\
\hline Wornom et al. [13], LES & 1.22 & 0.20 \\
\hline Nguyen and Nguyen [14], DES & 1.133 & 0.200 \\
\hline Gopalkrishnan [15], Exp & 1.186 & 0.193 \\
\hline Norberg [16], Exp & - & 0.202 \\
\hline Present study, URANS, 2D & 1.19 & 0.21 \\
\hline
\end{tabular}

The analysis of the test case allows selecting the optimal turbulence model, boundary conditions and computational cell size.

The model validation was implemented by comparing the numerical results with the experimental drag force values, $C_{d}$ the natural frequency of the test rod in the flow, $f_{n}$ and the flow-induced frequency $f_{t}$. The results obtained are summarised in Table 2. 
Table 2. Validation of the Full Numerical Model

\begin{tabular}{|l|c|c|c|}
\hline The study & $f_{n}$ & $f_{t}$ & $C_{d}$ \\
\hline Experiment (Upnere et al., 2020) & 58.50 & 27.30 & 0.346 \\
\hline CFD, 2D & 59.32 & 28.66 & 0.359 \\
\hline CFD, 3D & - & 28.56 & 0.352 \\
\hline
\end{tabular}

As shown in Table 2, the difference between the results of CFD and the experi-

\subsection{Dominant Frequencies}

The dominant frequencies were determined using the movement of the test cylinder and the calculated lift coefficient. The fast Fourier transform (FFT) and inverse mental measurements obtained is less than $5 \%$.

FFT method were applied to time series obtained from numerical simulations. The FFT approach was implemented in Matlab.

\section{SURROGATE MODELLING}

\subsection{Design of Experiments}

The selection of sampling points has a major effect on building a reliable surrogate model. Space-filling designs become a primary consideration for the design of computer simulation experiments [1]. Spacefilling design allows selecting those sample points that are distributed within the entire domain.

In this study, the new class of experimental designs described in [8] is used. Unlike classical CCD designs, in this case, all design points are within the research domain. Orthogonal designs of this type are particularly suitable for use with orthogonal Legendre polynomials detailed in the next subsection. This approach allows avoiding problems related to the optimal selection of significant terms, for example, in cases where the number of points is not much larger than the total number of possible terms. The following constraints were used to develop the experimental design [8]: 1) all experimental points were located in the unit cube $[-1,1]^{l}$; 2) designs had central symmetry, axial symmetry and 90-degree rotation symmetry properties; 3) designs were invariant to the permutation of input variables; 4) designs gave replications of centre point; 5) designs were orthogonal; 6) designs were D-optimal.

Figure 2 shows the 13-point orthogonal design used in the study to create a surrogate model with mass $m=[0.2765 ; 0.3253]$ and stiffness coefficient $k=[15725 ; 18500]$ as factors.

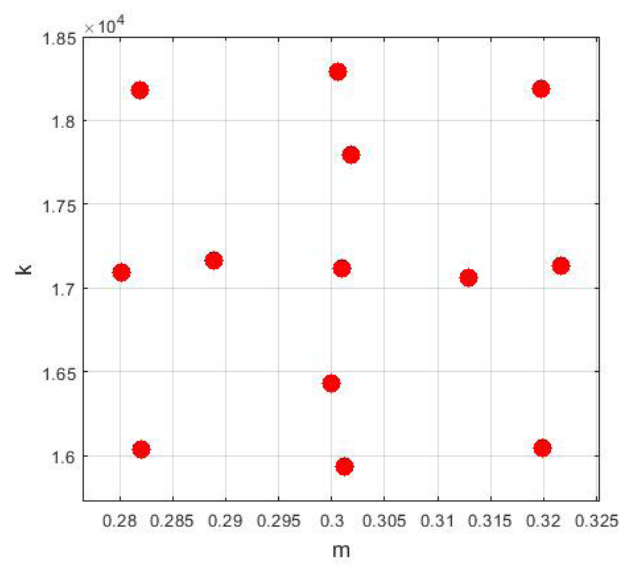

Fig. 2. The two factors (mass $m$ and stiffness coefficient $k$ ) orthogonal design with 13 points. 


\subsection{Response Surface Method}

Polynomial approximations produce surrogate models using low-order polynomials in a relatively small region of parameter space [2]. If the response function to be studied is expected to be linear, the firstorder polynomial approximation can be used, defined as [17]:

$\hat{y}=\beta_{0}+\beta_{1} x_{1}+\beta_{2} x_{2}+\cdots+\beta_{n} x_{n}+\varepsilon$,

where $\hat{y}$ is the system response, $x_{\mathrm{i}}, i=0,1, \ldots, n$, are independent variables, the parameters $\beta_{i}$ are called the regression coefficients, $\varepsilon$ represents the noise or error observed in the response $y$ assumed to be independent and normally distributed with mean zero and constant variance $\sigma^{2}$.

If the system under investigation is nonlinear, a higher-order polynomial should be used. For example, in the general case, a second-order polynomial regression model with $n$ simulation inputs is as shown below:

$\hat{y}=\beta_{0}+\sum_{i=1}^{n} \beta_{i} x_{i}+\sum_{i=1}^{n} \beta_{i i} x_{i}^{2}+\sum_{i=1}^{n-1} \sum_{j=i+1}^{n} \beta_{i j} x_{i} x_{j}+\varepsilon$.

Regression coefficients $\beta$ in Eq. (2) are estimated applying the least-squares criterion

$\hat{y}=\left(X^{\prime} X\right)^{-1} X^{\prime} w$,

where $w$ denotes the $n$-dimensional vector with the simulation outputs. The leastsquares estimator $\hat{\beta}$ exists if and only if the inverse of $X^{\prime} X$ exists; e.g., $\hat{\beta}$ exists if $X$ is orthogonal [18]. The method of the least squares returns the vector of the regression model parameters, which minimizes the sum of squared errors between the predictions of the regression model and the values of the true function.

\subsection{Legendre Polynomial Approximation}

The Legendre polynomials $P_{i}(x)$ are defined over $x \in[-1 ; 1]$ by the recurrence relation as [19]:

$$
\left\{\begin{array}{c}
P_{0}(x)=1 \\
P_{1}(x)=x \\
(i+1) P_{i+1}(x)=(2 i+1) x P(x)-i P_{i-1}(x), i-1,2, \ldots
\end{array}\right.
$$

The classic and discrete Legendre polynomials are orthogonal. In this study, orthonormal Legendre polynomials are used, which means that all terms must be multi- plied by $\sqrt{\frac{2 i+1}{2}}$. For onedimensional problem the first 4 orthonormal polynomials are: 


$$
\left\{\begin{array}{c}
P_{0}(x)=\sqrt{\frac{1}{2}} \\
P_{1}(x)=\sqrt{\frac{3}{2} x} \\
P_{2}(x)=\sqrt{\frac{5}{2}}\left(3 x^{2}-1\right) / 2 \\
P_{3}(x)=\sqrt{\frac{7}{2}}\left(5 x^{3}-3 x\right) / 2
\end{array} .\right.
$$

The multivariable polynomial terms are the product of single variable polynomials and they are orthonormal. The property of orthonormality allows for optimal selection of significant terms in the approximation polynomial function [8]. This is useful in cases where multivariate high order polynomials are used since in such situations the number of terms is rapidly increased and makes the application more difficult. The cross-validation criterion is used to decide whether to eliminate or keep the term with a small Euclid norm [8]:

$$
\left\|P_{i}(x)\right\|=\sqrt{\int_{-1}^{1} P_{i}^{2}(x) d x}=\sqrt{\frac{2}{2 i+1}} .
$$

In surrogate models, assessment methods should be applied to check if the developed models are adequate. The selection of approximations here is based on strategies described in the next subsection.

\subsection{Surrogate Model Adequacy and Fitting}

Myers et al. [1] define two steps for checking the response surface: 1) to examine the fitted model to ensure that it provides an adequate approximation to the true system and 2) to verify that none of the leastsquares regression assumptions is violated.

One of the techniques for checking model adequacy is residual analysis. In the context of surrogate modelling, the residual is defined as

$e_{i}=y_{i}-\hat{y}_{i}, i=1,2, \ldots, n$.

In the plot of residuals $e_{\mathrm{i}}$ versus the predicted response $\hat{y}_{i}$, the residuals should scatter randomly, suggesting that the variance of the original observations is constant for all values of $y$.

To estimate the generalization error, leave- $k$-out cross-validations commonly used. The cross-validation error can be used for surrogate model parameter estimation, model selection and validation when it is too costly to employ a separate validation data set [3]. Based on the study of Meckesheimer et al. [20], the value of $k=1$ is recommended for providing a prediction error estimate for low-order polynomials, and for Kriging surrogate models it is rec- ommended to select $k$ as a function of the fitting design size, for example, $k=0.1 \mathrm{~N}$ or $k=\sqrt{N}$. In this study, the relative crossvalidation error is expressed as:

$\sigma_{c r \%}=\frac{\sqrt{\frac{1}{n} \sum_{i=1}^{n}\left(\hat{y}_{-i}-y_{i}\right)^{2}}}{\sqrt{\frac{1}{n-1} \sum_{i=1}^{n}\left(y_{i}-\bar{y}\right)^{2}}} 100 \%$,

where $\hat{y}_{-i}$ denotes the prediction of the response using the surrogate model created without the point $i(i=1,2, \ldots, n)$ and $\bar{y}$ is the average value of $y$.

For regression models, $\mathrm{R}^{2}$-type metrics ( $\mathrm{R}^{2}$ is the coefficient of determination) are the commonly used goodness-of-fit measure. An adjusted coefficient of determination $\mathrm{R}_{\text {adj }}^{2}$ is used to avoid the fact that adding a variable to the model always increases the value of $\mathrm{R}^{2}$. Pearson's $\chi^{2}$ test is another commonly used metric. The $\chi^{2}$ test is a goodness-of-fit test to see whether the data are in concordance with the null hypothesis $H_{0}$ [7]. Pearson's $\chi^{2}$ test indicates whether the frequency distribution of specific events observed in the sample corresponds to a theoretical distribution. 
CFD simulations are used to obtain the response values in the sampling points. The values from the high-fidelity model

\subsection{Forward Model}

Based on the results of the laboratory experiments described in Upnere et al. [9], the frequency ratio between the natural frequency of the rod in the flow $f_{\mathrm{n}}$ and the flow-induced frequency $f_{\mathrm{t}}$ is selected as a response for the surrogate modelling.

As the design of experiments (see Fig. 2) is orthogonal, Legendre polynomials can be used as an approximation. The transformation from physical units to the unit cube $[-1 ; 1]^{2}$ is as follows:

$x_{1}=40.983896062695 \mathrm{~m}-12.332054325265$, $x_{2}=0.0007207207207207 \mathrm{k}-12.333333333333$, are applied to construct an approximation model that would indirectly detect a change in the mass of the rod.

where $m=[0.2765,0.3253]$ and $k=[15725$, 18500].

Analysis of the significance of polynomial terms based on Euclid norm in Eq. (6) shows that the coefficient per term does not depend on the number of terms used for approximation. This is due to the orthogonality of the design of experiments. From the relative cross-validation error depending on the number of terms of the Legendre polynomials presented in Fig. 3a, it follows that half of the polynomial terms can be discarded without affecting the outcome.

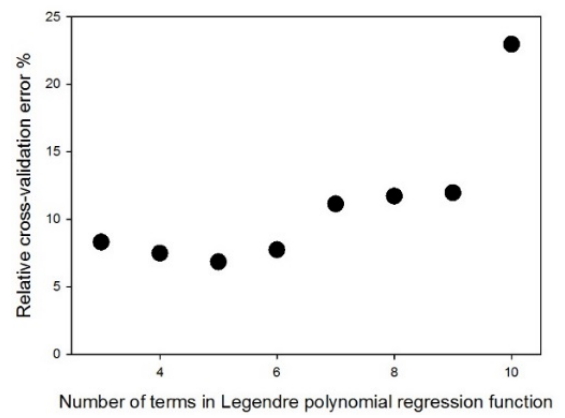

(a)

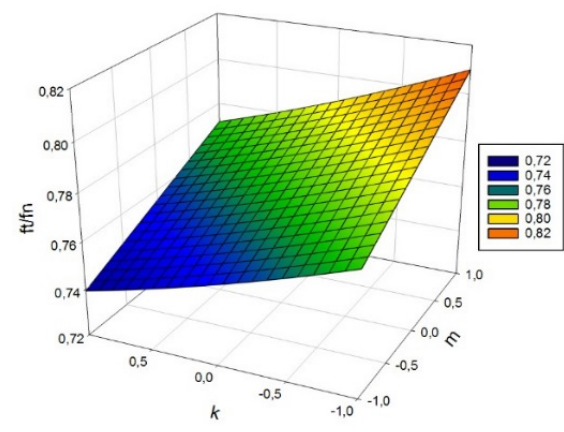

(b)

Fig. 3. Legendre polynomials: (a) the relative cross-validation error depending on the number of orthogonal terms; (b) response surface of 5-term Legendre polynomials in the unit cube.

The response surface of the 5-terms polynomial model in the unit cube is shown in Fig. $3 \mathrm{~b}$ and the corresponding Legendre polynomial can be described as follows:

$$
\begin{aligned}
& \hat{y}\left(x_{1}, x_{2}\right)=1.544039 P_{0} P_{0}+0.020323 P_{0} P_{1}\left(x_{1}\right)-0.020521 P_{0} P_{1}\left(x_{2}\right)+ \\
& 0.001028 P_{0} P_{2}\left(x_{2}\right)-0.000967 P_{1}\left(x_{1}\right) P_{1}\left(x_{2}\right) .
\end{aligned}
$$


In addition to Legendre polynomials, the first, second and third-order polynomials are investigated. The comparative results of the model quality metrics are summarised in Table 3.

Table 3. Approximation Quality Indicators of the Forward Model

\begin{tabular}{|l|c|c|}
\hline Approximation & $\sigma_{\mathrm{cr} \%}$ & $\mathrm{R}^{2}{ }_{\text {adj }}$ \\
\hline First-order & 8.31 & 0.996 \\
\hline Second-order & 8.16 & 0.998 \\
\hline Third-order & 22.96 & 0.998 \\
\hline Legendre & 6.85 & 0.998 \\
\hline
\end{tabular}

Table 3 shows that there is a small difference between the first- and second-order polynomial approximation which gives $8.31 \%$ and $8.16 \%$ cross-validation error, respectively. From Fig. 3a and Table 3 it follows that the lowest error $-6.85 \%$ is obtained by 5 -term Legendre polynomials.

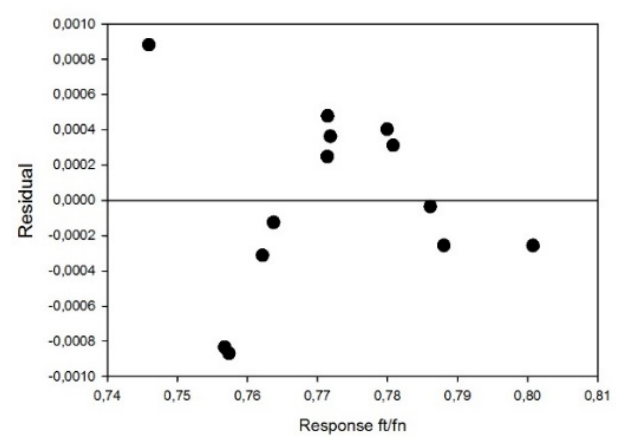

(a)
The adequacy of the model is also confirmed by plots of residuals that are randomly scattered (see Fig. 4). The residual versus response $f_{\mathrm{t}} / f_{\mathrm{n}}$ is shown in Fig. $4 \mathrm{a}$. The residual values are not correlated with factors $x_{1}$ and $x_{2}$. The example of the residual versus input $x_{1}$ is presented in Fig. 4b.

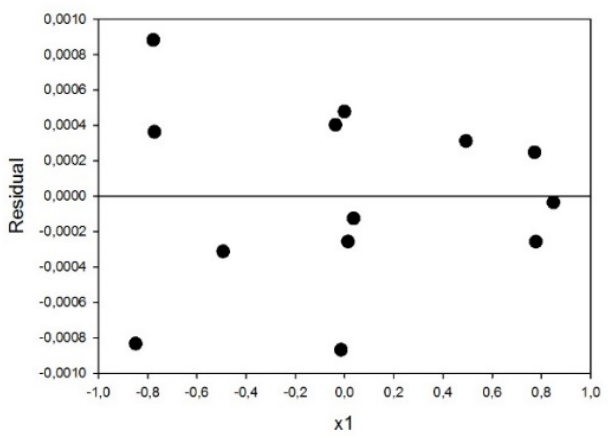

(b)

Fig. 4. The residual and regression line: (a) the residual values vs. response; (b) the residual vs. factor $x_{1}$.

\subsection{Inverse Model}

The inverse surrogate model for predicting changes of the oscillating cylinder mass was created using $f / f_{\mathrm{n}}$ and $k$ as factors. The formulas for transformation to unit cube for Legendre polynomials are:

$x_{1}=37.2562667089699 f_{t} / f_{n}-28: 8236008539$, $x_{2}=0.0008489240339426 \mathrm{k}-14: 52721253084$,

where $f_{\mathrm{t}} / f_{\mathrm{n}}=[0.746816665,0.800498909]$ and $k=[15934.5382978725$, $18290.4617021275]$.
As the design of experiments is not orthogonal, the coefficients for the terms of the Legendre polynomials vary depending on the number of terms. Therefore, the use of Legendre polynomials is not as effective as in the forward model. The comparative results of the model quality metrics are summarised in Table 4. 
Table 4. Approximation Quality Indicators of the Inverse Model

\begin{tabular}{|l|c|c|}
\hline Approximation & $\sigma_{\mathrm{cr} \%}$ & $\mathrm{R}_{\text {adj }}^{2}$ \\
\hline First-order & 11.52 & 0.992 \\
\hline Second-order & 11.37 & 0.996 \\
\hline Third-order & 36.41 & 0.995 \\
\hline Legendre & 8.95 & 0.997 \\
\hline
\end{tabular}

As can be seen from Table 4, there is only one method of approximation, for which the crossvalidation error is less than $10 \%$, the 4-term Legendre polynomials. Therefore, the Legendre polynomials were selected for building the surrogate model. Figure 5 presents response surfaces of 4-term Legendre polynomials and the relative cross-validation error depending on the number of terms.

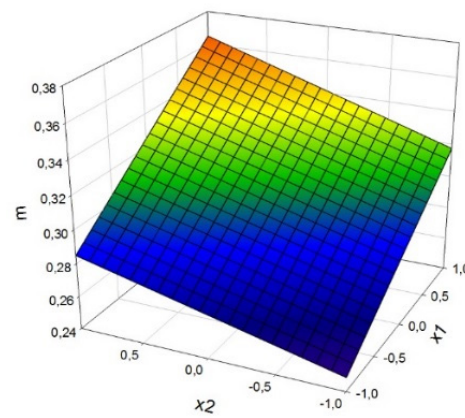

(a)

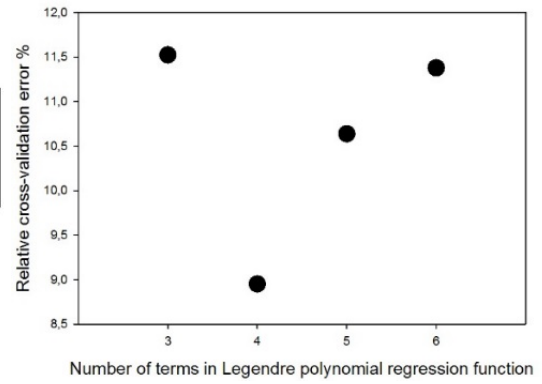

(b)

Fig. 5. Legendre polynomials of inverse surrogate model: (a) the response surface designed using 4 terms; (b) the relative cross-validation error vs. terms of the polynomial.

The residual graph shows the absence of correlation between the response and

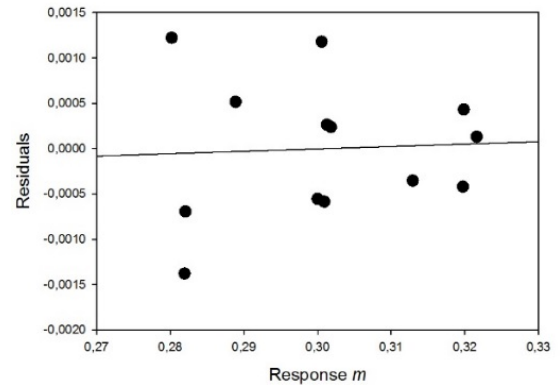

(a) residual values. Examples of residuals are shown in Fig. 6.

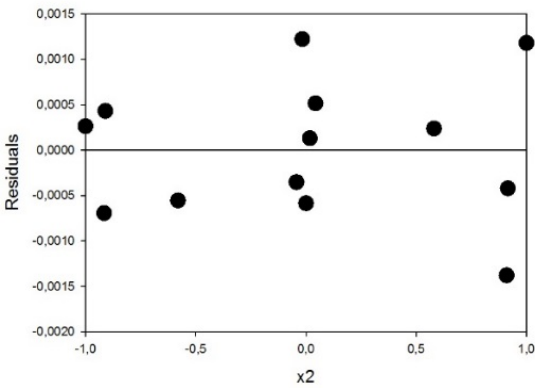

(b)

Fig. 6. Residual values and regression line of the inverse surrogate model: (a) the residual versus predicted response; (b) residual values versus input $x_{2}$.

The obtained model equation using 4-term Legendre polynomials is as follows:

$$
m\left(f_{t} / f_{n}\right)=-0.11026 f_{t} / f_{n}-4.97899 \cdot 10^{-5} k+8.74349 \cdot 10^{-5} f_{t} / f_{n} \cdot k+0.083652
$$


The inverse model verification was implemented by selecting specific points and comparing the results of the model and numerical experiments at these points. The comparison graph is shown in Fig. 7. The confidence interval $C I$ is determined to measure the uncertainty around the estimated values:

$C I=\bar{y} \pm z \cdot \frac{\sigma}{\sqrt{n}}$,

where $\sigma$ is standard deviation and for $95 \%$ $C I z=1.96$. The upper and the lower confidence bound can be seen in Fig. 7 with a dashed blue line.

From Fig. 7, it can be concluded that the resulting surrogate model can predict the behaviour of the analysed model system with high precision - when input factor values are changed within $15 \%$, the difference is less than $1.5 \%$.

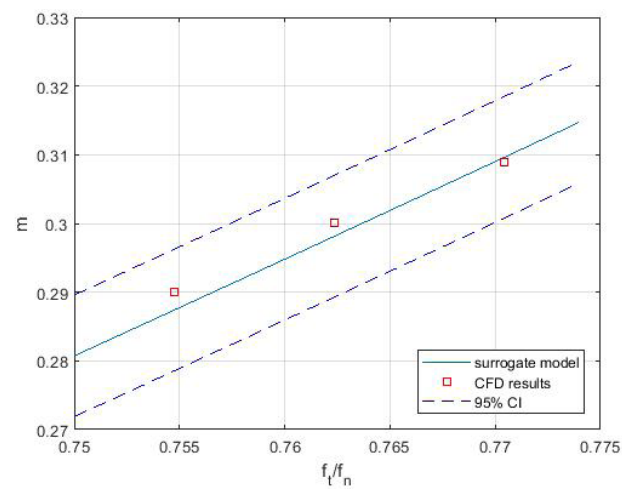

Fig. 7. Verification of the inverse surrogate model if the stiffness coefficient is $17600 \mathrm{~N} / \mathrm{m}$.

\section{CONCLUSIONS}

A methodology was developed for creating a surrogate model for the mass reduction analysis of the rod in the rod bundle inserted in the crossflow. The interaction between mass and stiffness as factors and frequency ratio as the response was applied to build the forward model. The inverse surrogate model was created using mass as a function of stiffness and frequency ratio.

The new third-order orthogonal design of experiments was used to define sample points. Orthogonality of the experimental design allowed for simple elimination of insignificant terms in Legendre polynomial approximations.

Four methods of approximation were investigated: first-, second- and third-order polynomials and Legendre polynomials. The most appropriate approximation for both the direct and inverse models for the problem under study was obtained using Legendre polynomials. The corresponding cross-validation errors were $6.85 \%$ and $8.95 \%$. Verification of the inverse model showed that the difference between the results of the CFD calculations and the predicted values of the surrogate model was less than $1.5 \%$.

The analysis carried out showed that the developed method of approximation and the obtained factors-response relationships had a good fit with numerical experiments. The results also demonstrated that the surrogate model could reliably replace the finite volume simulations and drastically reduce computational time. Therefore, the proposed methodology is useful in the monitoring of cooling systems as well as in the design process of such systems. 
The research has been partially conducted with the financial support of the Latvian Council of Science project "Creation of Design of Experiments and Metamodel- ing Methods for Optimization of Dynamics of Multibody 3D Systems Interacting with Bulk Solids and Fluids" (1zp-2018/2-0281).

\section{REFERENCES}

1. Myers, R. H., Montgomery, D. C., \& Anderson-Cook, C. M. (2016). Response surface methodology: Process and product optimization using designed experiments. New York: John Wiley \& Sons.

2. Khaledi, K., Miro, S., Konig, M., \& Schanz, T. (2014). Robust and Reliable Metamodels for Mechanized Tunnel Simulations. Computers and Geotechnics, 61, 1-12.

3. Forrester, A., \& Keane, A. (2009). Recent Advances in Surrogate-Based Optimization. Progress in Aerospace Science, 45 (1-3), 50-79.

4. Filho, U., Antunes, A., Bastos, S., \& Lyra, P. (2015). Minimization of Vortex Induced Vibrations Using Surrogate Based Optimization. Structural and Multidisciplinary Optimization, 52 (4), 717-735.

5. Banyay, G. A., Shields, M. D., \& Brigham, J. C. (2019). Efficient Global Sensitivity Analysis for Flow-Induced Vibration of a Nuclear Reactor Assembly Using Kriging Surrogates. Nuclear Engineering and Design, 341, 1-15.

6. Banyay, G. A., Shields, M. D., \& Brigham, J. C. (2020). Efficient Global Sensitivity Analysis of Structural Vibration for a Nuclear Reactor System Subject to Nonstationary Loading. Nuclear Engineering and Design, 361, 1-14.

7. Greenwood, P. E., \& Nikulin, M. S. (1996). A guide to chi-squared testing. New York: John Wiley \& Sons.

8. Auzins, J. (2014). High order orthogonal designs of experiments for metamodeling, identification and optimization of mechanical systems, in Oñate, E., Oliver,
J. \& Huerta, A. (eds.), 11th World Congress on Computational Mechanics, 5th European Conference on Computational Mechanics, and 6th European Conference on Computational Fluid Dynamics, 31903201.

9. Upnere, S., Jekabsons, N., Dementjevs, S., \& Wohlmuther, M. (2020). Effects of Variable Parameters on the Behaviour of the Single Flexibly-Mounted Rod in a CloselyPacked Array. Journal of Vibroengineering, 22, 1-20.

10. Roache, P. J. (1998). Verification and validation in computational science and engineering. New Mexico: Hermosa Publishers.

11. Khan, N., Ibrahim, Z., Nguyen, L., Javed, M., \& Jameel, M. (2017). Numerical Investigation of the Vortex-Induced Vibration of an Elastically Mounted Circular Cylinder at High Reynolds Number $\left(\mathrm{Re}=10^{4}\right)$ and Low Mass Ratio Using the RANS Code. PLoS ONE, 12 (10), 1-17.

12. Dong, S., \& Karniadakis, G. (2005). DNS of Flow Past a Stationary and Oscillating Cylinder at $\mathrm{Re}=10000$. Journal of Fluids and Structures, 20 (4), 519-531.

13. Wornom, S., Ouvrard, H., Salvetti, M., Koobus, B., \& Dervieux, A. (2011). Variational Multiscale Large-Eddy Simulations of the Flow past a Circular Cylinder: Reynolds Number Effects. Computers \& Fluids, 47 (1), 44-50.

14. Nguyen, V.-T., \& Nguyen, H. (2016). Detached Eddy Simulations of Flow Induced Vibrations of Circular Cylinders at High Reynolds Numbers. Journal of Fluids and Structures, 63, 103-119. 
15. Gopalkrishnan, R. (1993). Vortex-Induced Forces on Oscillating Bluff Cylinders. $\mathrm{PhD}$ Thesis, Massachusetts Institute of Technology.

16. Norberg, C. (2003). Fluctuating Lift on a Circular Cylinder: Review and New Measurements. Journal of Fluids and Structures, 17 (1), 57-96.

17. Montgomery, D. (2017). Design and analysis of experiments. New York: John Wiley \& Sons.

18. Kleijnen, J. P. C. (2016). Regression and Kriging Metamodels with their Experimental Designs in Simulation: A Review. European Journal of Operational Research, 000, 1-6.
19. Feng, X., Zhang, Y., \& Wu, J. (2018). Interval Analysis Method Based on Legendre Polynomial Approximation for Uncertain Multibody Systems. Advances in Engineering Software, 121, 223-234.

20. Meckesheimer, M., Booker, A., Barton, R., \& Simpson, T. (2002). Computationally Inexpensive Metamodel Assessment Strategies. AIAA Journal, 40 (10), 2053 2060. 